\title{
Toeplitz Geometries over Vector Spaces Induced from his 4-order Symmetric Matrices
}

\author{
Grozio Stanilov \\ Faculty of Mathematics and Informatics Sofia University “St. Kliment Ohridski” 1164 Sofia, Bulgaria \\ *Corresponding Author: stanilov@uni-sofia.bg
}

Copyright (C) 2014 Horizon Research Publishing All rights reserved.

\begin{abstract}
We establish that a subset of symmetric no degenerated 4- order Toeplitz matrices is a group and introduce the corresponding geometry called Toeplitz geometry. We introduce also the notion of induced Toeplitz geometry. Essentially we show that in these geometries any two vectors have some absolute invariants and defining the notion of plane we find also some absolute invariants of pairs consisting from vectors and planes. For all investigations we apply only the computer system Maple.
\end{abstract}

Keywords Toeplitz Matrix, Toeplitz Geometry, Induced Toeplitz Geometry, Invariant

AMS Classification: 51B25, 51N30, 20B30, 20C40

\section{Introduction}

We include the package with(LInearAlgebra):

The notion of 4-order symmetric Toeplitz matrix can be defined in the following way:

$\mathrm{V} 4:=<\mathrm{q}, \mathrm{r}, \mathrm{s}, \mathrm{t}>$ : with(LInearAlgebra):

T4:=ToeplitzMatrix(V4, 4, symmetric);

$$
T 4:=\left[\begin{array}{llll}
q & r & s & t \\
r & q & r & s \\
s & r & q & r \\
t & s & r & q
\end{array}\right]
$$

Consider another such matrix

$>\mathrm{U} 4:=\operatorname{eval}(\mathrm{T} 4,[\mathrm{q}=\mathrm{q} 1, \mathrm{r}=\mathrm{r} 1, \mathrm{~s}=\mathrm{s} 1, \mathrm{t}=\mathrm{t} 1])$;

$$
U 4:=\left[\begin{array}{llll}
q 1 & r 1 & s 1 & t 1 \\
r 1 & q 1 & r 1 & s 1 \\
s 1 & r 1 & q 1 & r 1 \\
t 1 & s 1 & r 1 & q 1
\end{array}\right]
$$

and multiply them m:=T4.U4 we easy establish that the product is again such matrix only if $t=r, t_{1}=r_{1}$. Then consider the subset of such no degenerated matrices with $t=r$.

$\mathrm{T} 41:=\operatorname{eval}(\mathrm{T} 4,[\mathrm{t}=\mathrm{r}])$;

$$
T_{41}=\left(\begin{array}{llll}
q & r & s & r \\
r & q & r & s \\
s & r & q & r \\
r & s & r & q
\end{array}\right)
$$

We easy establish that the inverse matrix of such matrix is again such matrix. Thus we can formulate the

THEOREM 1. The set of Toeplitz symmetric no degenerated matrices of type $T_{41}$ is a group.

This 3-parametrical group we denote by $\mathrm{Tg}_{3}$ and call 3-parametrical Toeplitz group. It arises a geometry, called Toeplitz geometry in 4-dimensional vector space denoted by $T G_{41}$. To find its fundamental transformations we consider the matricist

$$
c_{4}=\left(\begin{array}{c}
x \\
y \\
z \\
u
\end{array}\right), \quad C_{4}=\left(\begin{array}{c}
X_{41} \\
Y_{41} \\
Z_{41} \\
U_{41}
\end{array}\right),
$$

where

$$
\begin{aligned}
& X_{41}=q x+r y+s z+r u \\
& Y_{41}=r x+q y+r z+s u \\
& Z_{41}=s x+r y+q z+r u \\
& U_{41}=r x+s y+r z+q u
\end{aligned}
$$

The product $T_{41} c_{4}$ is exactly the matrix $C_{4}$. The formulas (2) represent a linear operator in the 4-dimensional vector space. The action is: $(x, y, z, u) \rightarrow\left(X_{41}, Y_{41}, Z_{41}, U_{41}\right)$. We accept (2) as fundamental transformations in the Toeplitz geometry $T G_{41}$. The considered set of linear operators (2) is a group of linear operators acting in the 4-dimensional vector space.

Together with the matrix $T_{41}$ we introduce the matrix 


$$
m_{3}=\left(\begin{array}{ccc}
q & 2 r & s \\
r & q+s & r \\
s & 2 r & q
\end{array}\right)
$$

called induced from $T_{41}$ matrix. It is easy to prove the following

THEOREM 2. The set of no degenerated matrices of type (3) is a group.

This group we denote by $i T g_{3}$ and call it induced Toeplitz group. It arises a geometry called induced Toeplitz geometry in 3-dimensional vector space and denoted by $I T G_{3}$. To find the fundamental transformations in this geometry we consider another matrix as $T_{41}$ :

$$
\begin{aligned}
\mathrm{p}:=\operatorname{eval}(\mathrm{T} 41,[\mathrm{q}=\mathrm{x}, \mathrm{r}=\mathrm{y}, \mathrm{s}=\mathrm{z}]) & \\
p & :=\left[\begin{array}{llll}
x & y & z & y \\
y & x & y & z \\
z & y & x & y \\
y & z & y & x
\end{array}\right]
\end{aligned}
$$

Their product $\mathrm{n}:=\mathrm{T} 41 . \mathrm{p}$ is of course such matrix with elements

$$
\mathrm{X}:=\mathrm{n}[1,1] ; \mathrm{Y}:=\mathrm{n}[1,2] ; \mathrm{Z}:=\mathrm{n}[1,3] \text {; }
$$

The set of formulas

$$
\begin{aligned}
& X=q x+2 r y+s z \\
& Y=r x+(q+s) y+r z \\
& Z=s x+2 r y+q z
\end{aligned}
$$

we accept as fundamental transformation in the induced Toeplitz geometry $\mathrm{ITG}_{3}$.Any such transformation acts in 3-dimensional vector space so: $(x, y, z) \rightarrow(X, Y, Z)$. The formulas (4) represent a linear operator. The set of all such linear operators (4) is a group of linear operators in 3-dimensional vector space.

We remark that the relations

$$
q=1, r=0, s=0
$$

represent the units for both groups.

From now on we begin to investigate both geometries.

\section{Base Facts in the Toeplitz Geometry}

\subsection{Invariants of Vectors in Toeplitz Geometry}

Using the fundamental formulas in this geometry we calculate easy:

factor $(\mathrm{X} 41+\mathrm{Y} 41+\mathrm{Z} 41+\mathrm{U} 41)$;

$(x+y+u+z)(q+s+2 r)$

factor(X41-Z41); $(x-z)(q-s)$

factor(X41+Y41-Z41-U41); $(x+y-z-u)(q-s)$

factor $(\mathrm{X} 41-\mathrm{Y} 41+\mathrm{Z} 41-\mathrm{U} 41)$;

$-(x-y-u+z)(-q-s+2 r)$

These results give us occasion to formulate the following assertions:

THEOREM 3.

1. For any vector $(x, y, z, u)$ in the Toeplitz geometry the expressions

(6) $x+y+z+u, x-z, x+y-z-u, x-y+z-u$

are relative invariants.

2. For any two vectors $\left(x_{1}, y_{1}, z_{1}, u_{1}\right),\left(x_{2}, y_{2}, z_{2}, u_{2}\right)$ the expressions.

$$
I_{12}=\frac{x_{1}+y_{1}+z_{1}+u_{1}}{x_{2}+y_{2}+z_{2}+u_{2}}, K_{12}=\frac{x_{1}-z_{1}}{x_{2}-z_{2}}, L_{12}=\frac{x_{1}+y_{1}-z_{1}-u_{1}}{x_{2}+y_{2}-z_{2}-u_{2}}, M_{12}=\frac{x_{1}-y_{1}+z_{1}-u_{1}}{x_{2}-y_{2}+z_{2}-u_{2}}
$$

are absolute invariants

3. The following four directions

$$
x+y+z+u=0, x-z=0, x+y-z-u=0, x-y+z-u=0
$$

are invariant directions in the 4-dimensional vector space.

Later we establish that these are not all invariant directions.

\subsection{Invariants of Hyper Planes (3-Dimensional Affine Manifolds)}

Such notion can be defined by the relation

$$
h=a x+b y+c z+d u+e,
$$

where $a, b, c, d, e$ are fixed numbers. At first we want to find how it is transformed by the equations (2). For the sake of convenience we instead of $X_{41}, Y_{41}, Z_{41}, U_{41}$ write in (2) simple correspondently $X, Y, Z, U$. Then we solve the system: solve $\left(\left\{X=\mathrm{q}^{*} \mathrm{x}+\mathrm{r}^{*} \mathrm{y}+\mathrm{s}^{*} \mathrm{z}+\mathrm{r}^{*} \mathrm{u}, \mathrm{Y}=\mathrm{r}^{*} \mathrm{x}+\mathrm{q}^{*} \mathrm{y}+\mathrm{r}^{*} \mathrm{z}+\mathrm{s}^{*} \mathrm{u}, \mathrm{Z}=\mathrm{s}^{*} \mathrm{x}+\mathrm{r}^{*} \mathrm{y}+\mathrm{q}^{*} \mathrm{z}+\mathrm{r}^{*} \mathrm{u}, \mathrm{U}=\mathrm{r}^{*} \mathrm{x}+\mathrm{s}^{*} \mathrm{y}+\mathrm{r}^{*} \mathrm{z}+\mathrm{q} * \mathrm{u}\right\},\{\mathrm{x}, \mathrm{y}, \mathrm{z}, \mathrm{u}\}\right)$; 


$$
\begin{aligned}
\{z= & -\frac{2 r^{2} X-2 r^{2} Z+q^{2} Z+r s U-q r Y+s r Y-r q U-q s X+q s Z-s^{2} X}{-4 r^{2} s+q s^{2}+s^{3}-q^{3}-q^{2} s+4 q r^{2}}, \\
u & =-\frac{q^{2} U-q r X+q s U-q s Y-q r Z-s^{2} Y+s r X+r s Z-2 r^{2} U+2 r^{2} Y}{-4 r^{2} s+q s^{2}+s^{3}-q^{3}-q^{2} s+4 q r^{2}}, \\
y & =\frac{-2 r^{2} U+2 r^{2} Y+q r X+q r Z-r s Z-s r X+q s U-q^{2} Y+s^{2} U-q s Y}{-4 r^{2} s+q s^{2}+s^{3}-q^{3}-q^{2} s+4 q r^{2}}, \\
x & \left.=\frac{-s r Y-q s X-2 r^{2} Z+q r Y+2 r^{2} X-r s U+r q U+q s Z+s^{2} Z-q^{2} X}{-4 r^{2} s+q s^{2}+s^{3}-q^{3}-q^{2} s+4 q r^{2}}\right\}
\end{aligned}
$$

We put these solutions in (9):

$\mathrm{g}:=$ eval $\left(\mathrm{a}^{*} \mathrm{x}+\mathrm{b} * \mathrm{y}+\mathrm{c}^{*} \mathrm{z}+\mathrm{d}^{*} \mathrm{u}+\mathrm{e},\left[\mathrm{z}=-1 /\left(-4 * \mathrm{r}^{\wedge} 2 * \mathrm{~s}+\mathrm{q}^{*} \mathrm{~s}^{\wedge} 2+\mathrm{s}^{\wedge} 3-\mathrm{q} \wedge 3-\mathrm{q}^{\wedge} 2 * \mathrm{~s}+4 * \mathrm{q} * \mathrm{r}^{\wedge} 2\right) *\left(2 * \mathrm{r}^{\wedge} 2 * \mathrm{X}-2 * \mathrm{r}^{\wedge} 2 * \mathrm{Z}+\mathrm{q}^{\wedge} 2 * \mathrm{Z}+\mathrm{r}^{*} \mathrm{~s}^{*} \mathrm{U}-\mathrm{q} * \mathrm{r}^{*}\right.\right.\right.$ $\left.\mathrm{Y}+\mathrm{s} * \mathrm{r} * \mathrm{Y}-\mathrm{r} * \mathrm{q} * \mathrm{U}-\mathrm{q} * \mathrm{~s} * \mathrm{X}+\mathrm{q} * \mathrm{~s} * \mathrm{Z}-\mathrm{s}^{\wedge} 2 * \mathrm{X}\right), \mathrm{u}=-\left(\mathrm{q} \wedge 2 * \mathrm{U}-\mathrm{q} * \mathrm{r} * \mathrm{X}+\mathrm{q} * \mathrm{~s} * \mathrm{U}-\mathrm{q} * \mathrm{~s}^{*} \mathrm{Y}-\mathrm{q} * \mathrm{r} * \mathrm{Z}-\mathrm{s} \wedge 2 * \mathrm{Y}+\mathrm{s} * \mathrm{r} * \mathrm{X}+\mathrm{r} * \mathrm{~s} * \mathrm{Z}-2 * \mathrm{r}^{\wedge} 2 * \mathrm{U}+2 * \mathrm{r}^{\wedge} 2 * \mathrm{Y}\right)$ $/\left(-4 * \mathrm{r}^{\wedge} 2 * \mathrm{~s}+\mathrm{q}^{*} \mathrm{~s}^{\wedge} 2+\mathrm{s}^{\wedge} 3-\mathrm{q}^{\wedge} 3-\mathrm{q} \wedge 2 * \mathrm{~s}+4 * \mathrm{q} * \mathrm{r}^{\wedge} 2\right), \mathrm{y}=\left(-2 * \mathrm{r}^{\wedge} 2 * \mathrm{U}+2 * \mathrm{r}^{\wedge} 2 * \mathrm{Y}+\mathrm{q} * \mathrm{r}^{*} \mathrm{X}+\mathrm{q} * \mathrm{r} * \mathrm{Z}-\mathrm{r} * \mathrm{~s} * \mathrm{Z}-\mathrm{s} * \mathrm{r} * \mathrm{X}+\mathrm{q} * \mathrm{~s}^{*} \mathrm{U}-\mathrm{q}^{\wedge} 2 * \mathrm{Y}+\mathrm{s}^{\wedge} 2 * \mathrm{U}-\mathrm{q}^{*}\right.$ $\left.\mathrm{s}^{*} \mathrm{Y}\right) /\left(-4 * \mathrm{r}^{\wedge} 2 * \mathrm{~s}+\mathrm{q} * \mathrm{~s}^{\wedge} 2+\mathrm{s}^{\wedge} 3-\mathrm{q} \wedge 3-\mathrm{q} \wedge 2 * \mathrm{~s}+4 * \mathrm{q} * \mathrm{r}^{\wedge} 2\right), \mathrm{x}=\left(-\mathrm{s} * \mathrm{r}^{*} \mathrm{Y}-\mathrm{q} * \mathrm{~s} * \mathrm{X}-2 * \mathrm{r}{ }^{\wedge} 2 * \mathrm{Z}+\mathrm{q} * \mathrm{r} * \mathrm{Y}+2 * \mathrm{r} \wedge 2 * \mathrm{X}-\mathrm{r} * \mathrm{~s} * \mathrm{U}+\mathrm{r} * \mathrm{q} * \mathrm{U}+\mathrm{q} * \mathrm{~s} * \mathrm{Z}+\mathrm{s}^{\wedge} 2 *\right.$ $\left.\left.\left.\mathrm{Z}-\mathrm{q}^{\wedge} 2 * \mathrm{X}\right) /\left(-4 * \mathrm{r}^{\wedge} 2 * \mathrm{~s}+\mathrm{q}^{*} \mathrm{~s}^{\wedge} 2+\mathrm{s}^{\wedge} 3-\mathrm{q}^{\wedge} 3-\mathrm{q}^{\wedge} 2 * \mathrm{~s}+4 * \mathrm{q} * \mathrm{r}^{\wedge} 2\right)\right]\right)$;

we get

$$
\begin{aligned}
g:= & \frac{a\left(-s r Y-q s X-2 r^{2} Z+q r Y+2 r^{2} X-r s U+r q U+q s Z+s^{2} Z-q^{2} X\right)}{-4 r^{2} s+q s^{2}+s^{3}-q^{3}-q^{2} s+4 q r^{2}} \\
& +\frac{b\left(-2 r^{2} U+2 r^{2} Y+q r X+q r Z-r s Z-s r X+q s U-q^{2} Y+s^{2} U-q s Y\right)}{-4 r^{2} s+q s^{2}+s^{3}-q^{3}-q^{2} s+4 q r^{2}} \\
& -\frac{c\left(2 r^{2} X-2 r^{2} Z+q^{2} Z+r s U-q r Y+s r Y-r q U-q s X+q s Z-s^{2} X\right)}{-4 r^{2} s+q s^{2}+s^{3}-q^{3}-q^{2} s+4 q r^{2}} \\
& -\frac{d\left(q^{2} U-q r X+q s U-q s Y-q r Z-s^{2} Y+s r X+r s Z-2 r^{2} U+2 r^{2} Y\right)}{-4 r^{2} s+q s^{2}+s^{3}-q^{3}-q^{2} s+4 q r^{2}}+e
\end{aligned}
$$

Now we apply the operation collect $(\mathrm{g},[\mathrm{X}, \mathrm{Y}, \mathrm{Z}, \mathrm{U}])$ and get

$$
\begin{aligned}
& \left(\frac{a\left(-q^{2}-q s+2 r^{2}\right)}{-4 r^{2} s+q s^{2}+s^{3}-q^{3}-q^{2} s+4 q r^{2}}+\frac{b(q r-s r)}{-4 r^{2} s+q s^{2}+s^{3}-q^{3}-q^{2} s+4 q r^{2}}\right. \\
& \left.\quad-\frac{c\left(2 r^{2}-q s-s^{2}\right)}{-4 r^{2} s+q s^{2}+s^{3}-q^{3}-q^{2} s+4 q r^{2}}-\frac{d(-q r+s r)}{-4 r^{2} s+q s^{2}+s^{3}-q^{3}-q^{2} s+4 q r^{2}}\right) X \\
& \quad+\left(\frac{a(q r-s r)}{-4 r^{2} s+q s^{2}+s^{3}-q^{3}-q^{2} s+4 q r^{2}}+\frac{b\left(-q^{2}-q s+2 r^{2}\right)}{-4 r^{2} s+q s^{2}+s^{3}-q^{3}-q^{2} s+4 q r^{2}}\right. \\
& \left.-\frac{c(-q r+s r)}{-4 r^{2} s+q s^{2}+s^{3}-q^{3}-q^{2} s+4 q r^{2}}-\frac{d\left(2 r^{2}-q s-s^{2}\right)}{-4 r^{2} s+q s^{2}+s^{3}-q^{3}-q^{2} s+4 q r^{2}}\right) Y \\
& +\left(\frac{a\left(-2 r^{2}+q s+s^{2}\right)}{-4 r^{2} s+q s^{2}+s^{3}-q^{3}-q^{2} s+4 q r^{2}}+\frac{b(q r-s r)}{-4 r^{2} s+q s^{2}+s^{3}-q^{3}-q^{2} s+4 q r^{2}}\right. \\
& \left.-\frac{c\left(q^{2}+q s-2 r^{2}\right)}{-4 r^{2} s+q s^{2}+s^{3}-q^{3}-q^{2} s+4 q r^{2}}-\frac{d(-q r+s r)}{-4 r^{2} s+q s^{2}+s^{3}-q^{3}-q^{2} s+4 q r^{2}}\right) Z \\
& +\left(\frac{b\left(-2 r^{2}+q s+s^{2}\right)}{-4 r^{2} s+q s^{2}+s^{3}-q^{3}-q^{2} s+4 q r^{2}}-\frac{c(-q r+s r)}{-4 r^{2} s+q s^{2}+s^{3}-q^{3}-q^{2} s+4 q r^{2}}\right. \\
& \left.-\frac{d\left(q^{2}+q s-2 r^{2}\right)}{-4 r^{2} s+q s^{2}+s^{3}-q^{3}-q^{2} s+4 q r^{2}}+\frac{a(q r-s r)}{-4 r^{2} s+q s^{2}+s^{3}-q^{3}-q^{2} s+4 q r^{2}}\right) U
\end{aligned}
$$

Denote:

$>P:=$ factor $\left(a^{*}\left(-q^{\wedge} 2-q^{*} s+2 * r^{\wedge} 2\right) /\left(-4 * r^{\wedge} 2 * s+q^{*} s^{\wedge} 2+s^{\wedge} 3-q^{\wedge} 3-q^{\wedge} 2 * s+4 * q^{*} r^{\wedge} 2\right)+b^{*}\left(q^{*} r-s^{*} r\right) /\left(-4 * r^{\wedge} 2 * s+q^{*} s^{\wedge} 2+s^{\wedge} 3-q^{\wedge} 3-q^{\wedge} 2 *\right.\right.$ 
$\left.\mathrm{s}+4 * \mathrm{q}^{*} \mathrm{r}^{\wedge} 2\right)-\mathrm{c} /\left(-4 * \mathrm{r}^{\wedge} 2 * \mathrm{~s}+\mathrm{q}^{*} \mathrm{~s}^{\wedge} 2+\mathrm{s}^{\wedge} 3-\mathrm{q}^{\wedge} 3-\mathrm{q}^{\wedge} 2{ }^{*} \mathrm{~s}+4 * \mathrm{q} * \mathrm{r}^{\wedge} 2\right) *\left(2 * \mathrm{r}^{\wedge} 2-\mathrm{q}^{*} \mathrm{~s}-\mathrm{s}^{\wedge} 2\right)-\mathrm{d} *\left(-\mathrm{q} * \mathrm{r}+\mathrm{s}^{*} \mathrm{r}\right) /\left(-4 * \mathrm{r}^{\wedge} 2 * \mathrm{~s}+\mathrm{q}^{*} \mathrm{~s}^{\wedge} 2+\mathrm{s}^{\wedge} 3-\mathrm{q}^{\wedge} 3-\mathrm{q}^{\wedge} 2 * \mathrm{~s}^{*}+\right.$ $\left.\left.4 * \mathrm{q}^{*} \mathrm{r}^{\wedge} 2\right)\right)$; $\mathrm{Q}:=$ factor $\left(\mathrm{a}^{*}\left(\mathrm{q}^{*} \mathrm{r}-\mathrm{s}^{*} \mathrm{r}\right) /\left(-4 * \mathrm{r}^{\wedge} 2 * \mathrm{~s}+\mathrm{q}^{*} \mathrm{~s}^{\wedge} 2+\mathrm{s}^{\wedge} 3-\mathrm{q}^{\wedge} 3-\mathrm{q}^{\wedge} 2 * \mathrm{~s}+4 * \mathrm{q}^{*} \mathrm{r}^{\wedge} 2\right)+\mathrm{b}^{*}\left(-\mathrm{q}^{\wedge} 2-\mathrm{q}^{*} \mathrm{~s}+2 * \mathrm{r}^{\wedge} 2\right) /\left(-4 * \mathrm{r}^{\wedge} 2{ }^{*} \mathrm{~s}+\mathrm{q}^{*} \mathrm{~s}^{\wedge} 2+\mathrm{s}^{\wedge} 3-\mathrm{q}^{\wedge}\right.\right.$ $\left.3-\mathrm{q}^{\wedge} 2 * \mathrm{~s}+4 * \mathrm{q}^{*} \mathrm{r}^{\wedge} 2\right)-\mathrm{c} /\left(-4 * \mathrm{r}^{\wedge} 2 * \mathrm{~s}+\mathrm{q}^{*} \mathrm{~s}^{\wedge} 2+\mathrm{s}^{\wedge} 3-\mathrm{q}^{\wedge} 3-\mathrm{q}^{\wedge} 2 * \mathrm{~s}+4 * \mathrm{q}^{*} \mathrm{r}^{\wedge} 2\right) *\left(-\mathrm{q} * \mathrm{r}+\mathrm{s}^{*} \mathrm{r}\right)-\mathrm{d}^{*}\left(2 * \mathrm{r}^{\wedge} 2-\mathrm{q}^{*} \mathrm{~s}-\mathrm{s}^{\wedge} 2\right) /\left(-4 * \mathrm{r}^{\wedge} 2 * \mathrm{~s}^{*}+\mathrm{q}^{*} \mathrm{~s}^{\wedge} 2+\mathrm{s}^{\wedge} 3-\mathrm{q}^{\wedge} 3-\right.$ $\left.\left.\mathrm{q}^{\wedge} 2 * \mathrm{~s}+4 * \mathrm{q}^{*} \mathrm{r}^{\wedge} 2\right)\right) ; \mathrm{R}:=$ factor $\left(\mathrm{a}^{*}\left(-2 * \mathrm{r}^{\wedge} 2+\mathrm{q}^{*} \mathrm{~s}+\mathrm{s}^{\wedge} 2\right) /\left(-4 * \mathrm{r}^{\wedge} 2 * \mathrm{~s}+\mathrm{q}^{*} \mathrm{~s}^{\wedge} 2+\mathrm{s}^{\wedge} 3-\mathrm{q}^{\wedge} 3-\mathrm{q}^{\wedge} 2 * \mathrm{~s}+4 * \mathrm{q}^{*} \mathrm{r}^{\wedge} 2\right)+\mathrm{b}^{*}\left(\mathrm{q} * \mathrm{r}-\mathrm{s}^{*} \mathrm{r}\right) /\left(-4 * \mathrm{r}^{\wedge} 2{ }^{*} \mathrm{~s}+\mathrm{q}^{*} \mathrm{~s}^{\wedge} 2\right.\right.$ $\left.+\mathrm{s}^{\wedge} 3-\mathrm{q}^{\wedge} 3-\mathrm{q}^{\wedge} 2 * \mathrm{~s}+4 * \mathrm{q} * \mathrm{r}^{\wedge} 2\right)-\mathrm{c} /\left(-4 * \mathrm{r}^{\wedge} 2 * \mathrm{~s}+\mathrm{q}^{*} \mathrm{~s}^{\wedge} 2+\mathrm{s}^{\wedge} 3-\mathrm{q}^{\wedge} 3-\mathrm{q}^{\wedge} 2 * \mathrm{~s}+4 * \mathrm{q} * \mathrm{r}^{\wedge} 2\right) *\left(\mathrm{q}^{\wedge} 2+\mathrm{q}^{*} \mathrm{~s}-2 * \mathrm{r}^{\wedge} 2\right)-\mathrm{d} *\left(-\mathrm{q}^{*} \mathrm{r}+\mathrm{s}^{*} \mathrm{r}\right) /\left(-4 * \mathrm{r}^{\wedge} 2 * \mathrm{r}^{*}+\mathrm{q}^{*} \mathrm{~s}^{\wedge} 2+\right.$ $\left.\left.\mathrm{s}^{\wedge} 3-\mathrm{q}^{\wedge} 3-\mathrm{q}^{\wedge} 2 * \mathrm{~s}+4 * \mathrm{q}^{*} \mathrm{r}^{\wedge} 2\right)\right) ; \mathrm{S}:=$ factor $\left(\mathrm{b}^{*}\left(-2 * \mathrm{r}^{\wedge} 2+\mathrm{q}^{*} \mathrm{~s}+\mathrm{s}^{\wedge} 2\right) /\left(-4 * \mathrm{r}^{\wedge} 2 * \mathrm{~s}+\mathrm{q}^{*} \mathrm{~s}^{\wedge} 2+\mathrm{s}^{\wedge} 3-\mathrm{q}^{\wedge} 3-\mathrm{q}^{\wedge} 2 * \mathrm{~s}+4 * \mathrm{q}^{*} \mathrm{r}^{\wedge} 2\right)-\mathrm{c} /\left(-4 * \mathrm{r}^{\wedge} 2 * \mathrm{~s}^{*}+\mathrm{q}^{*} \mathrm{~s}^{\wedge} 2+\mathrm{s}\right.\right.$ $\left.{ }^{\wedge} 3-\mathrm{q}^{\wedge} 3-\mathrm{q}^{\wedge} 2 * \mathrm{~s}+4 * \mathrm{q} * \mathrm{r}^{\wedge} 2\right) *\left(-\mathrm{q} * \mathrm{r}+\mathrm{s}^{*} \mathrm{r}\right)-\mathrm{d}^{*}\left(\mathrm{q}^{\wedge} 2+\mathrm{q} * \mathrm{~s}-2 * \mathrm{r}^{\wedge} 2\right) /\left(-4 * \mathrm{r}^{\wedge} 2 * \mathrm{~s}+\mathrm{q}^{*} \mathrm{~s}^{\wedge} 2+\mathrm{s}^{\wedge} 3-\mathrm{q}^{\wedge} 3-\mathrm{q}^{\wedge} 2 * \mathrm{~s}+4 * \mathrm{q} * \mathrm{r}^{\wedge} 2\right)+\mathrm{a} *\left(\mathrm{q} * \mathrm{r}-\mathrm{s}^{*} \mathrm{r}\right) /\left(-4 * \mathrm{r}^{\wedge} 2 * \mathrm{~s}\right.$ $\left.\left.+\mathrm{q}^{*} \mathrm{~s}^{\wedge} 2+\mathrm{s}^{\wedge} 3-\mathrm{q}^{\wedge} 3-\mathrm{q}^{\wedge} 2 * \mathrm{~s}+4 * \mathrm{q}^{*} \mathrm{r}^{\wedge} 2\right)\right)$

we have

$$
\begin{aligned}
P & :=\frac{-a q^{2}-a q s+2 a r^{2}+b r q-b r s-2 c r^{2}+c q s+c s^{2}+d r q-d r s}{(q-s)(q+s+2 r)(-q-s+2 r)} \\
Q & :=\frac{a r q-a r s-b q^{2}-b q s+2 b r^{2}+c r q-c r s-2 d r^{2}+d q s+d s^{2}}{(q-s)(q+s+2 r)(-q-s+2 r)} \\
R & :=-\frac{2 a r^{2}-a q s-a s^{2}-b r q+b r s+c q^{2}+c q s-2 c r^{2}-d r q+d r s}{(q-s)(q+s+2 r)(-q-s+2 r)} \\
S & :=-\frac{2 b r^{2}-b q s-b s^{2}-c r q+c r s+d q^{2}+d q s-2 d r^{2}-a r q+a r s}{(q-s)(q+s+2 r)(-q-s+2 r)}
\end{aligned}
$$

We establish at once:

factor $(\mathrm{P} * \mathrm{X}+\mathrm{Q} * \mathrm{Y}+\mathrm{R} * \mathrm{Z}+\mathrm{S} * \mathrm{U}+\mathrm{e})$;

$$
a x+b y+c z+d u+e
$$

Thus we can formulate the following

\section{THEOREM 4.}

1. The hyper plane

$$
h=a x+b y+c z+d u+e
$$

is transformed in the Toeplitz geometry into

$$
H=P X+Q Y+R Z+S U+e
$$

More precisely it holds

$$
P X+Q Y+R Z+S U+e=a x+b y+c z+d u+e,
$$

where $P, Q, R, S$ are given over.

2. Any vector $(x, y, z, u)$. and any hyper plane $(a, b, c, d)$ in the Toeplitz geometry have the invariant

$$
\operatorname{Inv}(\text { vector, hyperplane })=a x+b y+c z+d u \text {. }
$$

\subsection{Invariants of Two Hyper Planes}

Considering the Map $(a, b, c, d) \rightarrow(P, Q, R, S)$ We Calculate

$>$ factor(P-R); $-\frac{-c+a}{-q+s}$

$>$ factor $(\mathrm{Q}-\mathrm{S}) ;-\frac{-d+b}{-q+s}$

$>$ factor $(\mathrm{P}+\mathrm{R}+\mathrm{Q}+\mathrm{S}) ; \frac{d+c+a+b}{s+q+2 r}$

These results give us occasion to formulate

\section{THEOREM 5.}

1. For any hyper plane the following expressions

$$
a-c, b-d, a+b+c+d
$$

are relative invariants. 
2. For any two hyper planes $\left(a_{1}, b_{1}, c_{1}, d_{1}\right),\left(a_{2}, b_{2}, c_{2}, d_{2}\right)$ the expressions

$$
\frac{a_{1}-c_{1}}{a_{2}-c_{2}}, \frac{b_{1}-d_{1}}{b_{2}-d_{2}}, \frac{a_{1}+b_{1}+c_{1}+d_{1}}{a_{2}+b_{2}+c_{2}+d_{2}}
$$

are absolute invariants.

Now we want to find all invariant directions. To do this we solve the system:

$>\operatorname{solve}(\{\mathrm{P}=1 * \mathrm{a}, \mathrm{Q}=1 * \mathrm{~b}, \mathrm{R}=1 * \mathrm{c}, \mathrm{S}=1 * \mathrm{~d}\},\{\mathrm{a}, \mathrm{b}, \mathrm{c}, \mathrm{d}, 1\})$;

$$
\begin{gathered}
\left\{b=b, c=c, d=-b, a=-c, l=\frac{1}{q-s}\right\},\{b=0, d=0, a=0, l=l, c=0\}, \\
\left\{l=-\frac{1}{-q-s+2 r}, b=b, c=-b, d=b, a=-b\right\}, \\
\left\{b=b, c=b, a=b, d=b, l=\frac{1}{q+s+2 r}\right\}
\end{gathered}
$$

So we have found the following hyper planes:

$$
\begin{gathered}
e 11:=-c(x-z)+b(y-u)+e, e 22:=-b(x-y-u+z)+e \\
e 33:=b(x+y+u+z)+e
\end{gathered}
$$

Thus we can formulate

THEOREM 6. In the Toeplitz geometry all invariant directions are given by the relations :

$$
c(x-z)=b(y-u), x+y+z+u=0, x-y+z-u=0
$$

where $b, c$ are arbitrary numbers.

\subsubsection{2-Dimensional Planes (2-Dimensional Affine Manifolds)}

Such manifolds can be determined by two equations of the type (9). In general case they can be substituted by the equations of the form

$$
x=a_{1} z+b_{1} u+c_{1}, y=a_{2} z+b_{2} u+c_{2} .
$$

Here we shall be briefly. We bring here the solutions of the corresponding system. We get

$$
X=A_{1} Z+B_{1} U+C_{1}, Y=A_{2} Z+B_{2} U+C_{2}
$$

Where

$$
\begin{aligned}
A 1:= & \left(-r^{2} b 1 a 2+r^{2} b 2 a 1-b 2 s^{2}-a 1 q^{2}+a 1 r^{2}-a 2 q r+a 2 s r-b 1 s r+r^{2}+b 1 q r\right. \\
& \left.+b 1 a 2 q s-a 1 q b 2 s+b 2 r^{2}-q s\right) /\left(r^{2} b 2 a 1-r^{2} b 1 a 2+r^{2}+b 2 r^{2}+a 1 r^{2}\right. \\
& \left.-b 1 q r+b 1 s r+a 2 s r-a 2 q r-b 2 q s-a 1 q s-q^{2}-a 1 s^{2} b 2+b 1 a 2 s^{2}\right) \\
B 1: & =(q-s)(-b 1 q-r b 1 a 2-r b 2+r a 1 b 2-r+a 1 r-b 1 s) /\left(r^{2} b 2 a 1-r^{2} b 1 a 2\right. \\
& +r^{2}+b 2 r^{2}+a 1 r^{2}-b 1 q r+b 1 s r+a 2 s r-a 2 q r-b 2 q s-a 1 q s-q^{2} \\
& \left.-a 1 s^{2} b 2+b 1 a 2 s^{2}\right) \\
C 1: & (q-s)\left(-c 1 q^{2}+q b 1 c 2 s-r c 2 q-q b 2 c 1 s-q r a 2 c 1+q r a 1 c 2-q c 1 s\right. \\
& -r a 1 c 2 s+r s c 2+2 r^{2} b 2 c 1+2 c 1 r^{2}-2 r^{2} b 1 c 2+r a 2 s c 1+b 1 c 2 s^{2} \\
& \left.-b 2 c 1 s^{2}\right) /\left(r^{2} b 2 a 1-r^{2} b 1 a 2+r^{2}+b 2 r^{2}+a 1 r^{2}-b 1 q r+b 1 s r+a 2 s r\right. \\
& \left.-a 2 q r-b 2 q s-a 1 q s-q^{2}-a 1 s^{2} b 2+b 1 a 2 s^{2}\right) \\
A 2:= & (q-s)(-a 2 q-r b 1 a 2+r a 1 b 2-a 1 r-r+r b 2-a 2 s) /\left(r^{2} b 2 a 1-r^{2} b 1 a 2\right. \\
& +r^{2}+b 2 r^{2}+a 1 r^{2}-b 1 q r+b 1 s r+a 2 s r-a 2 q r-b 2 q s-a 1 q s-q^{2} \\
& \left.-a 1 s^{2} b 2+b 1 a 2 s^{2}\right)
\end{aligned}
$$




$$
\begin{aligned}
B 2:= & \left(-a 2 s r+a 1 r^{2}-b 2 q^{2}+b 1 a 2 q s-a 1 q b 2 s-a 1 s^{2}+b 2 r^{2}+a 2 q r+r^{2}\right. \\
& \left.-r^{2} b 1 a 2+r^{2} b 2 a 1-b 1 q r+b 1 s r-q s\right) /\left(r^{2} b 2 a 1-r^{2} b 1 a 2+r^{2}+b 2 r^{2}\right. \\
& +a 1 r^{2}-b 1 q r+b 1 s r+a 2 s r-a 2 q r-b 2 q s-a 1 q s-q^{2}-a 1 s^{2} b 2+b 1 a 2 s^{2} \\
&
\end{aligned}
$$

In this way it is proved

THEOREM 7. In the Toeplitz geometry $T G_{41}$ 2-dimensional plane

$$
x=a_{1} z+b_{1} u+c_{1}, y=a_{2} z+b_{2} u+c_{2}
$$

is transformed into the 2-dimensional plane

$$
X=A_{1} Z+B_{1} U+C_{1}, Y=A_{2} Z+B_{2} U+C_{2}
$$

where the coefficients $A_{1}, B_{1}, C_{1} ; A_{2}, B_{2}, C_{2}$ are given above.

We state now the question about the invariant (double) 2-dimensional plane. To do this we solve the system: solve $(\{\mathrm{A} 1=\mathrm{a} 1, \mathrm{~B} 1=\mathrm{b} 1, \mathrm{C} 1=\mathrm{c} 1, \mathrm{~A} 2=\mathrm{a} 2, \mathrm{~B} 2=\mathrm{b} 2, \mathrm{C} 2=\mathrm{c} 2\},\{\mathrm{a} 1, \mathrm{~b} 1, \mathrm{c} 1, \mathrm{a} 2, \mathrm{~b} 2, \mathrm{c} 2\})$;

$$
\begin{aligned}
\{a 1=-b 2, b 1=-1-b 2, a 2=b 2-1, c 1=0, c 2=0, b 2=b 2\}, & \\
& \{a 1=-b 2, b 1=1+b 2, a 2=-b 2+1, c 1=0, c 2=0, b 2=b 2\}, \\
& \{b 2=-1, c 1=0, a 2=0, b 1=0, c 2=0, a 1=-1\}, \\
& \{a 1=1, c 1=0, b 2=1, a 2=0, b 1=0, c 2=0\}
\end{aligned}
$$

Using these solutions we can formulate

THEOREM 8. In the Toeplitz geometry $T G_{41}$ all 2-dimensional invariant (double) planes are the following:

1. $x+u=-b(z+u), y+z=b(z+u)$

2. $x-u=b(-z+u), y-z=b(-z+u)$

3. $x+z=0, y+u=0$

4. $x-z=0, y-u=0$

where $b$ is arbitrary number.

As an application of the previous formulas we calculate

$>$ factor(A1+A2-B1-B2);

$$
\begin{gathered}
(-q+s)(-a 2+b 2+b 1-a 1)(s+2 r+q) /\left(-r^{2} b 2 a 1+r^{2} b 1 a 2-r^{2}-b 2 r^{2}-a 1 r^{2}\right. \\
\left.+b 1 q r-b 1 s r-a 2 s r+a 2 q r+b 2 q s+a 1 q s+q^{2}+a 1 s^{2} b 2-b 1 a 2 s^{2}\right)
\end{gathered}
$$

This result gives us occasion to formulate the following

LEMMA1. For any 2-dimnsional plane in the Toeplitz geometry the expression

$$
a_{1}+a_{2}-b_{1}-b_{2}
$$

is a relative invariant.

\subsection{Invariant (Double) Straight Lines}

A straight line can be determined by the equations

$$
x=a 1 u+a 2, y=b 1 u+b 2, z=c 1 u+c 2
$$

Using our method in the previous points we can establish 
THEOREM 9. In the Toeplitz geometry the straight line (20) is transformed into the straight line

$$
X=A_{1} U+A_{2}, Y=B_{1} U+B_{2}, Z=C_{1} U+C_{2}
$$

where

$$
\begin{gathered}
A 1:=\frac{b 1 r+r+c 1 s+a 1 q}{q+a 1 r+b 1 s+c 1 r} \\
A 2:=\left(-b 1 a 2 r^{2}+c 1 r^{2} b 2-b 1 c 2 r^{2}-c 2 r^{2}+a 1 r^{2} b 2-a 2 r^{2}+r b 2 q-s r c 1 a 2\right. \\
+s r a 1 c 2-r a 1 c 2 q+r c 1 q a 2-r b 2 s+b 1 s a 2 q-a 1 s b 2 q+c 2 q s+s^{2} b 1 c 2 \\
\left.-s^{2} c 1 b 2+q^{2} a 2\right) /(q+a 1 r+b 1 s+c 1 r) \\
B 1:=-\frac{-c 1 r-b 1 q-a 1 r-s}{q+a 1 r+b 1 s+c 1 r} \\
B 2:=-\frac{(s-q)(b 2 s-r b 1 c 2+r a 2+r c 1 b 2-r b 1 a 2+r c 2+r a 1 b 2+b 2 q)}{q+a 1 r+b 1 s+c 1 r} \\
C 1:=\frac{b 1 r+a 1 s+r+c 1 q}{q+a 1 r+b 1 s+c 1 r} \\
C 2:=\left(b 1 s^{2} a 2+b 1 c 2 q s-b 1 c 2 r^{2}-b 1 a 2 r^{2}-a 1 s^{2} b 2+q a 2 s+s r c 1 a 2-r b 2 s\right. \\
-s r a 1 c 2-s c 1 b 2 q+c 1 r^{2} b 2+r a 1 c 2 q-c 2 r^{2}+a 1 r^{2} b 2+r b 2 q+c 2 q^{2} \\
\left.-r c 1 q a 2-a 2 r^{2}\right) /(q+a 1 r+b 1 s+c 1 r)
\end{gathered}
$$

Now we can solve the problem about the invariance of the straight lines. To do this we solve the system: solve $(\{\mathrm{A} 1=\mathrm{a} 1, \mathrm{~A} 2=\mathrm{a} 2, \mathrm{~B} 1=\mathrm{b} 1, \mathrm{~B} 2=\mathrm{b} 2, \mathrm{C} 1=\mathrm{c} 1, \mathrm{C} 2=\mathrm{c} 2\},\{\mathrm{a} 1, \mathrm{a} 2, \mathrm{~b} 1, \mathrm{~b} 2, \mathrm{c} 1, \mathrm{c} 2\})$;

$$
\begin{array}{r}
\{b 1=-1, a 2=0, c 2=0, c 1=c 1, a 1=-c 1, b 2=0\}, \\
\quad\{a 2=0, a 1=1, c 2=0, c 1=1, b 1=1, b 2=0\}, \\
\quad\{a 2=0, c 2=0, a 1=-1, b 1=1, c 1=-1, b 2=0\}
\end{array}
$$

THEOREM 10. In the Toeplitz geometry $T G_{41}$ exactly the following straight lines are invariant (double):

1.

$$
\begin{array}{ll}
\text { 1. } & x=-c u, y=-u, z=c u \\
\text { 2. } & x=u, y=u, z=u \\
\text { 3. } & x=-u, y=u, z=-u,
\end{array}
$$

where $\mathrm{c}$ is an arbitrary number.

After calculation

$>$ factor(A1-C1); $-\frac{(-q+s)(-c 1+a 1)}{q+a 1 r+b 1 s+c 1 r}$

We can formulate the following

LEMMA 2

1. In the Toplitz geometry any straight line (20) has the relative invariant

$$
\mathrm{a}_{1}-c_{1}
$$

2. Ih the Toeplitz geometry any two straight lines

$$
\begin{aligned}
& x=a_{1} u+a_{2}, y=b_{1} u+b_{2}, z=c_{1} u+c_{2} \\
& x=a_{10} u+a_{20}, y=b_{10} u+b_{20}, z=c_{10} u+c_{20}
\end{aligned}
$$

have the absolute invariant

$$
\frac{a_{1}-c_{1}}{a_{10}-c_{10}} \text {. }
$$




\section{Base Facts in the Induced Toeplitz Geometry}

\subsection{Invariants of Vectors in the Induced Toeplitz Geometry}

Using the fundamental formulas (4) in this geometry we calculate easy:

$>$ factor $(\mathrm{X}-\mathrm{Z}) ;-(s-q)(x-z)$

$>$ factor $(\mathrm{X}+\mathrm{Z}+2 * \mathrm{Y}) ;(x+z+2 y)(s+q+2 r)$

$>$ factor $(\mathrm{X}+\mathrm{Z}-2 * \mathrm{Y}) ;-(x+z-2 y)(-s-q+2 r)$

Thus we can formulate

THEOREM 11

1. For any vector $(x, y, z)$ in the induced Toeplitz geometry the expressions

$$
x-z, x+z+2 y, x+z-2 y
$$

are relative invariants.

2. For any two vectors $\left(x_{1}, y_{1}, z_{1}\right),\left(x_{2}, y_{2}, z_{2}\right)$ the expressions

$$
k_{12}=\frac{x_{1}-z_{1}}{x_{2}-z_{2}}, l_{12}=\frac{x_{1}+z_{1}+2 y_{1}}{x_{2}+z_{2}+2 y_{2}}, m_{12}=\frac{x_{1}+z_{1}-2 y_{1}}{x_{2}+z_{2}-2 y_{2}}
$$

are absolute invariants

3. The following four directions

$$
x-z=0, x+z+2 y=0, x+z-2 y=0
$$

are invariant directions in the 3-dimensional vector space.

Later we establish that these are all invariant directions.

\subsection{Invariant of Vector and Plane (2-Dimensional Affine Manifolds)}

Such notion can be defined by the relation

$$
e=a x+b y+c z+d
$$

where $a, b, c, d$ are fixed numbers. At first we want to find how it is transformed by the equations (4). At first we solve the system:

system: $\operatorname{solve}(\{X=\mathrm{q} * \mathrm{x}+2 * \mathrm{r} * \mathrm{y}+\mathrm{s} * \mathrm{z}, \mathrm{Y}=\mathrm{r} * \mathrm{x}+(\mathrm{s}+\mathrm{q}) * \mathrm{y}+\mathrm{r} * \mathrm{z}, \mathrm{Z}=\mathrm{s} * \mathrm{x}+2 * \mathrm{r} * \mathrm{y}+\mathrm{q} * \mathrm{z}\},\{\mathrm{x}, \mathrm{y}, \mathrm{z}\}) ;$ system

$$
\begin{aligned}
\{y & =\frac{-q Y-s Y+r Z+r X}{-q^{2}-2 q s-s^{2}+4 r^{2}}, \\
x & =\frac{-X q^{2}-q s X+2 r^{2} X+2 q r Y-2 r^{2} Z+q s Z-2 s r Y+Z s^{2}}{\left(-q^{2}-2 q s-s^{2}+4 r^{2}\right)(q-s)}, \\
z & \left.=-\frac{Z q^{2}-2 q r Y-q s X+q s Z+2 r^{2} X-2 r^{2} Z-s^{2} X+2 s r Y}{\left(-q^{2}-2 q s-s^{2}+4 r^{2}\right)(q-s)}\right\}
\end{aligned}
$$

Then we put them in (26):

$>\mathrm{f}:=\operatorname{simplify}\left(\right.$ eval $\left(\mathrm{e},\left[\mathrm{x}=\left(-\mathrm{X}^{*} \mathrm{q} \wedge 2-\mathrm{q} * \mathrm{~s} * \mathrm{X}+2 * \mathrm{r}^{\wedge} 2 * \mathrm{X}+2 * \mathrm{q} * \mathrm{r} * \mathrm{Y}-2 * \mathrm{r}^{\wedge} 2 * \mathrm{Z}+\mathrm{q} * \mathrm{~s} * \mathrm{Z}-2 * \mathrm{~s}^{*} \mathrm{r}^{*} \mathrm{Y}+\mathrm{Z} * \mathrm{~s}^{\wedge} 2\right) /\left(-\mathrm{q} \wedge 2-2 * \mathrm{q}^{*} \mathrm{~s}-\mathrm{s}^{\wedge} 2+4 * \mathrm{r}^{\wedge} 2\right) /(\mathrm{q}-\right.\right.$ s), $\mathrm{Z}=-\left(\mathrm{Z} * \mathrm{q}^{\wedge} 2-2 * \mathrm{q} * \mathrm{r} * \mathrm{Y}-\mathrm{q}^{*} \mathrm{~s}^{*} \mathrm{X}+\mathrm{q} * \mathrm{~s} * \mathrm{Z}+2 * \mathrm{r}^{\wedge} 2 * \mathrm{X}-2 * \mathrm{r}^{\wedge} 2 * \mathrm{Z}-\mathrm{s}^{\wedge} 2 * \mathrm{X}+2 * \mathrm{~s}^{*} \mathrm{r}^{*} \mathrm{Y}\right) /\left(-\mathrm{q}^{\wedge} 2-2 * \mathrm{q} * \mathrm{~s}-\mathrm{s}^{\wedge} 2+4 * \mathrm{r}^{\wedge} 2\right) /(\mathrm{q}-\mathrm{s}), \mathrm{y}=\left(-\mathrm{q} * \mathrm{Y}-\mathrm{s}^{*} \mathrm{Y}+\mathrm{r}^{*}\right.$ $\left.\left.\left.\mathrm{Z}+\mathrm{r}^{*} \mathrm{X}\right) /\left(-\mathrm{q}^{\wedge} 2-2 * \mathrm{q}^{*} \mathrm{~s}-\mathrm{s}^{\wedge} 2+4 * \mathrm{r}^{\wedge} 2\right)\right]\right)$ );

$$
\begin{aligned}
f:= & \left(-4 d s r^{2}-a X q^{2}+2 a r^{2} X-2 a r^{2} Z+a Z s^{2}-b q^{2} Y+b s^{2} Y-c Z q^{2}-2 c r^{2} X\right. \\
& +2 c r^{2} Z+c s^{2} X-d s q^{2}+d s^{2} q+4 d q r^{2}-d q^{3}+d s^{3}-a q s X+2 a q r Y \\
& +a q s Z-2 a s r Y+b r q Z+b q r X-b s r Z-b s r X+2 c q r Y+c q s X \\
& -c q s Z-2 c s r Y) /\left(\left(-q^{2}-2 q s-s^{2}+4 r^{2}\right)(q-s)\right)
\end{aligned}
$$

After collection we have

$>$ collect(f, $[\mathrm{X}, \mathrm{Y}, \mathrm{Z}])$; 


$$
\begin{gathered}
\frac{\left(-a q^{2}+2 a r^{2}-2 c r^{2}+c s^{2}-a q s+b q r-b s r+c q s\right) X}{\left(-q^{2}-2 q s-s^{2}+4 r^{2}\right)(q-s)} \\
+\frac{\left(-b q^{2}+b s^{2}+2 a q r-2 a s r+2 c q r-2 c s r\right) Y}{\left(-q^{2}-2 q s-s^{2}+4 r^{2}\right)(q-s)} \\
+\frac{\left(-2 a r^{2}+a s^{2}-c q^{2}+2 c r^{2}+a q s+b q r-b s r-c q s\right) Z}{\left(-q^{2}-2 q s-s^{2}+4 r^{2}\right)(q-s)} \\
+\frac{-d q^{3}-d s q^{2}+d s^{2} q+d s^{3}+4 d q r^{2}-4 d s r^{2}}{\left(-q^{2}-2 q s-s^{2}+4 r^{2}\right)(q-s)}
\end{gathered}
$$

Denote

$>\mathrm{A}:=$ factor $\left(\mathrm{a}^{*}\left(-\mathrm{q}^{\wedge} 2-\mathrm{s}^{*} \mathrm{q}+2 * \mathrm{r}^{\wedge} 2\right) /\left(-\mathrm{s}^{\wedge} 2-2 * \mathrm{~s}^{*} \mathrm{q}-\mathrm{q}^{\wedge} 2+4 * \mathrm{r}^{\wedge} 2\right) /(-\mathrm{s}+\mathrm{q})+\mathrm{b} * \mathrm{r} /\left(-\mathrm{s}^{\wedge} 2-2^{*} \mathrm{~s}^{*} \mathrm{q}-\mathrm{q}^{\wedge} 2+4 * \mathrm{r}^{\wedge} 2\right)-\mathrm{c}^{*}\left(-\mathrm{s}^{*} \mathrm{q}-\mathrm{s}^{\wedge} 2+2 * \mathrm{r}^{\wedge} 2\right) /\left(-\mathrm{s}^{\wedge} 2-2 *\right.\right.$ $\left.\left.\mathrm{s}^{*} \mathrm{q}-\mathrm{q}^{\wedge} 2+4 * \mathrm{r}^{\wedge} 2\right) /(-\mathrm{s}+\mathrm{q})\right) ; \mathrm{B}:=$ factor $\left(\mathrm{a}^{*}\left(2 * \mathrm{q}^{*} \mathrm{r}-2^{*} \mathrm{~s}^{*} \mathrm{r}\right) /\left(-\mathrm{s}^{\wedge} 2-2^{*} \mathrm{~s}^{*} \mathrm{q}-\mathrm{q}^{\wedge} 2+4 * \mathrm{r}^{\wedge} 2\right) /(-\mathrm{s}+\mathrm{q})+\mathrm{b}^{*}(-\mathrm{q}-\mathrm{s}) /\left(-\mathrm{s}^{\wedge} 2-2^{*} \mathrm{~s}^{*} \mathrm{q}-\mathrm{q}^{\wedge} 2+4 * \mathrm{r}^{\wedge} 2\right)-\mathrm{c}^{*}(2 *\right.$ $\left.\left.\mathrm{s}^{*} \mathrm{r}-2 * \mathrm{q} * \mathrm{r}\right) /\left(-\mathrm{s}^{\wedge} 2-2 * \mathrm{~s}^{*} \mathrm{q}-\mathrm{q}^{\wedge} 2+4 * \mathrm{r}^{\wedge} 2\right) /(-\mathrm{s}+\mathrm{q})\right) ; \mathrm{C}:=$ factor $\left(\mathrm{a}^{*}\left(\mathrm{~s}^{*} \mathrm{q}+\mathrm{s}^{\wedge} 2-2 * \mathrm{r}^{\wedge} 2\right) /\left(-\mathrm{s}^{\wedge} 2-2^{*} \mathrm{~s}^{*} \mathrm{q}-\mathrm{q}^{\wedge} 2+4 * \mathrm{r}^{\wedge} 2\right) /(-\mathrm{s}+\mathrm{q})+\mathrm{b}^{*} \mathrm{r} /\left(-\mathrm{s}^{\wedge} 2-2^{*} \mathrm{~s}^{*} \mathrm{q}-\right.\right.$ $\left.\left.\mathrm{q}^{\wedge} 2+4 * \mathrm{r}^{\wedge} 2\right)-\mathrm{c} *\left(\mathrm{q}^{\wedge} 2+\mathrm{s}^{*} \mathrm{q}-2 * \mathrm{r}^{\wedge} 2\right) /\left(-\mathrm{s}^{\wedge} 2-2 * \mathrm{~s}^{*} \mathrm{q}-\mathrm{q}^{\wedge} 2+4 * \mathrm{r}^{\wedge} 2\right) /(-\mathrm{s}+\mathrm{q})\right)$;

$$
\begin{gathered}
A:=\frac{-a q^{2}-a s q+2 a r^{2}-b r s+b r q+c s q+c s^{2}-2 c r^{2}}{(q+s+2 r)(-q-s+2 r)(-s+q)} \\
B:=\frac{2 a r-b q-b s+2 c r}{(q+s+2 r)(-q-s+2 r)} \\
C:=-\frac{-a s q-a s^{2}+2 a r^{2}+b r s-b r q+c q^{2}+c s q-2 c r^{2}}{(q+s+2 r)(-q-s+2 r)(-s+q)}
\end{gathered}
$$

Then we calculate

$>\operatorname{simplify}(\mathrm{A} * \mathrm{X}+\mathrm{B} * \mathrm{Y}+\mathrm{C} * \mathrm{Z}+\mathrm{d})$;

$$
d+c z+b y+a x
$$

Thus we have proved

THEOREM 12.

1. The plane

$$
e=a x+b y+c z+d
$$

is transformed in the induced Toeplitz geometry into

$$
E=A X+B Y+C Z+d
$$

More precisely it holds

$$
A X+B Y+C Z=a x+b y+c z
$$

where $A, B, C$ are given over.

2. Any vector $(x, y, z)$ and any plane $(a, b, c)$ have the invariant

$$
\operatorname{inv}(\text { vector }, \text { plane })=a x+b y+c z \text {. }
$$

Now we want to solve exactly the problem about the double planes in the induced Toeplitz geometry. To do this we solve the system:

$>\operatorname{solve}\left(\left\{\mathrm{A}=1^{*} \mathrm{a}, \mathrm{B}=\mathrm{l}^{*} \mathrm{~b}, \mathrm{C}=\mathrm{l}^{*} \mathrm{c}\right\},\{\mathrm{a}, \mathrm{b}, \mathrm{c}, 1\}\right)$;

$$
\begin{aligned}
& \left\{l=\frac{1}{-s+q}, b=0, a=-c, c=c\right\},\{c=0, b=0, a=0, l=l\}, \\
& \quad\left\{a=a, c=a, l=-\frac{1}{-q-s+2 r}, b=-2 a\right\},\left\{a=a, c=a, l=\frac{1}{q+s+2 r}, b=2 a\right\}
\end{aligned}
$$

From these solutions we can formulate

THEOREM 12. The directions 3 in THEOREM 11 are exactly all double directions.

Using the calculation 
$>$ factor(A-C); $\frac{a-c}{-S+q}$

we can formulate

Lemma 3

1. In the induced Toeplitz geometry any 2-dimensional plane (27) has the relative invariant

$$
a-c \text {. }
$$

2. Any two 2-dimensional planes $\left(a_{1}, b_{1}, c_{1}\right),\left(a_{2}, b_{2}, c_{2}\right)$ in the induced Toeplitz geometry have the absolute invariant

$$
\frac{a_{1}-c_{1}}{a_{2}-c_{2}}
$$

\subsection{Invariant (Double) Straight Lines in the Induced Toeplitz Geometry}

A Straight Line can be determined by the equations: and

$$
x=a_{1} z+a_{2}, y=b_{1} z+b_{2} .
$$

We have:

f1: $=$ eval $\left(-\mathrm{x}+\mathrm{a} 1 * \mathrm{z}+\mathrm{a} 2,\left[\mathrm{x}\left(-\mathrm{Z}^{*} \mathrm{~s}^{\wedge} 2-\mathrm{q} * \mathrm{Z} * \mathrm{~s}+2 * \mathrm{r}^{\wedge} 2 * \mathrm{Z}+\mathrm{X} * \mathrm{q} \wedge 2+\mathrm{q} * \mathrm{~s} * \mathrm{X}-2 * \mathrm{q} * \mathrm{r} * \mathrm{Y}+2 * \mathrm{~s} * \mathrm{r} * \mathrm{Y}-2 * \mathrm{r}^{\wedge} 2 * \mathrm{X}\right) /\left(\mathrm{s} \wedge 2+2 * \mathrm{~s} * \mathrm{q}+\mathrm{q} \wedge 2-4 * \mathrm{r}^{\wedge} 2\right) /(-\mathrm{s}\right.\right.$ $+\mathrm{q}), \mathrm{Z}=-\left(-\mathrm{Z} * \mathrm{q} \wedge 2+2 * \mathrm{q} * \mathrm{r} * \mathrm{Y}+\mathrm{q} * \mathrm{~s} * \mathrm{X}-\mathrm{q} * \mathrm{Z} * \mathrm{~s}+\mathrm{s}^{\wedge} 2 * \mathrm{X}-2 * \mathrm{r}^{\wedge} 2 * \mathrm{X}+2 * \mathrm{r}^{\wedge} 2 * \mathrm{Z}-2 * \mathrm{~s}^{*} \mathrm{r}^{*} \mathrm{Y}\right) /\left(\mathrm{s}^{\wedge} 2+2 * \mathrm{~s} * \mathrm{q}+\mathrm{q} \wedge 2-4 * \mathrm{r}^{\wedge} 2\right) /(-\mathrm{s}+\mathrm{q}), \mathrm{y}=-1 /\left(\mathrm{s}^{\wedge} 2+2\right.$ $* \mathrm{~s} * \mathrm{q}+\mathrm{q} \wedge 2-4 * \mathrm{r} \wedge 2) *(-\mathrm{s} * \mathrm{Y}-\mathrm{q} * \mathrm{Y}+\mathrm{r} * \mathrm{X}+\mathrm{r} * \mathrm{Z})]) ;$ system

$$
\begin{aligned}
f l:= & -\frac{-Z s^{2}-q Z s+2 r^{2} Z+X q^{2}+q s X-2 q r Y+2 s r Y-2 r^{2} X}{\left(s^{2}+2 s q+q^{2}-4 r^{2}\right)(-s+q)} \\
& -\frac{a l\left(-Z q^{2}+2 q r Y+q s X-q Z s+s^{2} X-2 r^{2} X+2 r^{2} Z-2 s r Y\right)}{\left(s^{2}+2 s q+q^{2}-4 r^{2}\right)(-s+q)}+a 2
\end{aligned}
$$

fl: $=$ eval $\left(-\mathrm{x}+\mathrm{a} 1 * \mathrm{z}+\mathrm{a} 2,\left[\mathrm{x}=\left(-\mathrm{Z}^{*} \mathrm{~s}^{\wedge} 2-\mathrm{q} * \mathrm{Z} * \mathrm{~s}+2 * \mathrm{r}^{\wedge} 2 * \mathrm{Z}+\mathrm{X} * \mathrm{q} \wedge 2+\mathrm{q} * \mathrm{~s} * \mathrm{X}-2 * \mathrm{q} * \mathrm{r}^{*} \mathrm{Y}+2 * \mathrm{~s} * \mathrm{r}^{*} \mathrm{Y}-2 * \mathrm{r}^{\wedge} 2 * \mathrm{X}\right) /\left(\mathrm{s} \wedge 2+2 * \mathrm{~s}^{*} \mathrm{q}+\mathrm{q}^{\wedge} 2-4 * \mathrm{r}^{\wedge} 2\right) /(-\right.\right.$ $\mathrm{s}+\mathrm{q}), \mathrm{Z}=-\left(-\mathrm{Z}^{*} \mathrm{q} \wedge 2+2 * \mathrm{q} * \mathrm{r}^{*} \mathrm{Y}+\mathrm{q}^{*} \mathrm{~s} * \mathrm{X}-\mathrm{q} * \mathrm{Z}^{*} \mathrm{~s}+\mathrm{s}^{\wedge} 2 * \mathrm{X}-2 * \mathrm{r}^{\wedge} 2 * \mathrm{X}+2 * \mathrm{r}^{\wedge} 2 * \mathrm{Z}-2 * \mathrm{~s}^{*} \mathrm{r}^{*} \mathrm{Y}\right) /\left(\mathrm{s}^{\wedge} 2+2 * \mathrm{~s}^{*} \mathrm{q}+\mathrm{q}^{\wedge} 2-4 * \mathrm{r}^{\wedge} 2\right) /(-\mathrm{s}+\mathrm{q}), \mathrm{y}=-1 /\left(\mathrm{s}^{\wedge} 2+\right.$ $\left.\left.\left.2 * \mathrm{~s} * \mathrm{q}+\mathrm{q} \wedge 2-4 * \mathrm{r}^{\wedge} 2\right) *(-\mathrm{s} * \mathrm{Y}-\mathrm{q} * \mathrm{Y}+\mathrm{r} * \mathrm{X}+\mathrm{r} * \mathrm{Z})\right]\right) ;$ system

$$
\begin{aligned}
f 2:= & \frac{-s Y-q Y+r X+r Z}{s^{2}+2 s q+q^{2}-4 r^{2}} \\
& -\frac{b 1\left(-Z q^{2}+2 q r Y+q s X-q Z s+s^{2} X-2 r^{2} X+2 r^{2} Z-2 s r Y\right)}{\left(s^{2}+2 s q+q^{2}-4 r^{2}\right)(-s+q)}+b 2
\end{aligned}
$$

solve( $\{\mathrm{f} 1, \mathrm{f} 2\},\{\mathrm{X}, \mathrm{Y}\})$;

$$
\begin{aligned}
\{X= & -\left(-a 2 q^{2}+2 q a 1 r b 2-2 q b 1 r a 2+2 r b 2 s-q a 1 Z-2 q r b 2-2 a 1 r b 2 s\right. \\
& \left.+2 b 1 r a 2 s-Z s-2 r b 1 Z+a 2 s^{2}\right) /(a 1 s+q+2 b 1 r), Y=(-b 1 q a 2 s+b 1 q Z \\
& +2 b 1 a 2 r^{2}-b 1 a 2 s^{2}+b 1 Z s+b 2 q^{2}+q r a 2+q a 1 b 2 s+q b 2 s-2 r^{2} a 1 b 2 \\
& \left.\left.-2 b 2 r^{2}+r a 1 Z-r a 2 s+r Z+a 1 b 2 s^{2}\right) /(a 1 s+q+2 b 1 r)\right\}
\end{aligned}
$$

$\mathrm{X}:=\operatorname{collect}\left(-1 /\left(\mathrm{a} 1 *_{\mathrm{s}}+\mathrm{q}+2 * \mathrm{~b} 1 * \mathrm{r}\right) *(-\mathrm{a} 2\right.$

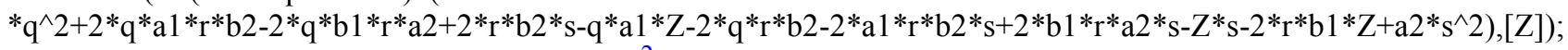

$$
X:=-\frac{(-a 1 q-2 b 1 r-s) Z}{a 1 s+q+2 b 1 r}-\frac{-a 2 q^{2}+2 q a 1 r b 2-2 q b 1 r a 2+2 r b 2 s-2 q r b 2-\varepsilon}{a 1 s+q+2 b 1 r}
$$

Denote:

A1: $=-1 /(\mathrm{a} 1 * \mathrm{~s}+\mathrm{q}+2 * \mathrm{~b} 1 * \mathrm{r}) *(-\mathrm{a} 1 * \mathrm{q}-2 * \mathrm{~b} 1 * \mathrm{r}-\mathrm{s}) ; \mathrm{A} 2:=-1 /(\mathrm{a} 1 * \mathrm{~s}+\mathrm{q}+2 * \mathrm{~b} 1 * \mathrm{r}) *\left(-\mathrm{a} 2 * \mathrm{q} \wedge 2+2 * \mathrm{q} * \mathrm{a} 1{ }^{\mathrm{r}} *_{\mathrm{b} 2}-2 * \mathrm{q} * \mathrm{~b} 1 * \mathrm{r} * \mathrm{a} 2+2 * \mathrm{r} * \mathrm{~b} 2 * \mathrm{~s}-2 * \mathrm{q}\right.$ $\left.*_{r} * b 2-2 * a 1 *_{r} * b 2 * s+2 * b 1 *_{r} * a 2 *_{s}+a 2 * s \wedge 2\right)$

$$
A 1:=-\frac{-a 1 q-2 b 1 r-s}{a 1 s+q+2 b 1 r}
$$




$$
A 2:=-\frac{-a 2 q^{2}+2 q a 1 r b 2-2 q b 1 r a 2+2 r b 2 s-2 q r b 2-2 a 1 r b 2 s+2 b 1 r a 2 s}{a 1 s+q+2 b 1 r}
$$

$\mathrm{Y}=$ collect( $\left(-\mathrm{b} 1{ }^{*} \mathrm{q} * \mathrm{a} 2 * \mathrm{~s}+\mathrm{b} 1 * \mathrm{q} * \mathrm{Z}+2 * \mathrm{~b} 1 * \mathrm{a} 2 * \mathrm{r}{ }^{\wedge} 2-\mathrm{b} 1 * \mathrm{a} 2 *{ }^{\wedge} 2+\mathrm{b} 1 * \mathrm{Z} * \mathrm{~s}+\mathrm{b} 2 * \mathrm{q} \wedge 2+\mathrm{q} * \mathrm{r} * \mathrm{a} 2+\mathrm{q} * \mathrm{a} 1 * \mathrm{~b} 2 * \mathrm{~s}+\mathrm{q} * \mathrm{~b} 2 * \mathrm{~s}-2 * \mathrm{r}^{\wedge} 2 * \mathrm{a} 1 * \mathrm{~b} 2-2 * \mathrm{~b}\right.$ $\left.\left.2 * \mathrm{r} \wedge 2+\mathrm{r} * \mathrm{a} 1 * \mathrm{Z}-\mathrm{r} * \mathrm{a} 2 * \mathrm{~s}+\mathrm{r} * \mathrm{Z}+\mathrm{a} 1{ }^{*} \mathrm{~b} 2{ }^{*} \mathrm{~s}^{\wedge} 2\right) /\left(\mathrm{a} 1{ }^{*} \mathrm{~s}+\mathrm{q}+2 * \mathrm{~b} 1{ }^{*} \mathrm{r}\right),[\mathrm{Z}]\right)$;

$$
\begin{gathered}
Y=\frac{(b 1 q+b 1 s+a 1 r+r) Z}{a 1 s+q+2 b 1 r}+\left(-b 1 q a 2 s+2 b 1 a 2 r^{2}-b 1 a 2 s^{2}+b 2 q^{2}+q r a 2\right. \\
\left.+q a 1 b 2 s+q b 2 s-2 r^{2} a 1 b 2-2 b 2 r^{2}-r a 2 s+a 1 b 2 s^{2}\right) /(a 1 s+q+2 b 1 r) \\
B 1:=\frac{b 1 q+b 1 s+a 1 r+r}{a 1 s+q+2 b 1 r} \\
B 2:=\left(-b 1 q a 2 s+2 b 1 a 2 r^{2}-b 1 a 2 s^{2}+b 2 q^{2}+q r a 2+q a 1 b 2 s+q b 2 s\right. \\
\left.-2 r^{2} a 1 b 2-2 b 2 r^{2}-r a 2 s+a 1 b 2 s^{2}\right) /(a 1 s+q+2 b 1 r)
\end{gathered}
$$

At the end we calculate:

$$
\begin{gathered}
\text { factor(eval } \left.\left(-\mathrm{X}+\mathrm{A} 1 * \mathrm{Z}+\mathrm{A} 2,\left[\mathrm{x}=\mathrm{a} 1 *_{\mathrm{z}}+\mathrm{a} 2, \mathrm{y}=\mathrm{b} 1 *_{\mathrm{z}}+\mathrm{b} 2\right]\right)\right) \\
0 \\
\text { factor(eval } \left.\left(-\mathrm{Y}+\mathrm{B} 1 * \mathrm{Z}+\mathrm{B} 2,\left[\mathrm{x}=\mathrm{a} 1 * \mathrm{z}+\mathrm{a} 2, \mathrm{y}=\mathrm{b} 1 *_{\mathrm{z}}+\mathrm{b} 2\right]\right)\right)
\end{gathered}
$$

Thus we have proved:

THEOREM 13. In the induced Toeplitz geometry a straight line

$$
x=a_{1} z+a_{2}, y=b_{1} z+b_{2}
$$

is transformed into straight line

$$
X=A_{1} Z+A_{2}, Y=B_{1} Z+B_{2}
$$

where the coefficients are given over.

Now we can find all invariant (double) straight lines. To do this we solve the system:

$>\operatorname{solve}(\{\mathrm{A} 1=\mathrm{a} 1, \mathrm{~A} 2=\mathrm{a} 2, \mathrm{~B} 1=\mathrm{b} 1, \mathrm{~B} 2=\mathrm{b} 2\},\{\mathrm{a} 1, \mathrm{a} 2, \mathrm{~b} 1, \mathrm{~b} 2\})$;

$$
\begin{aligned}
& \{a 1=1, b 2=0, a 2=0, b 1=1\},\{a 1=1, b 2=0, a 2=0, b 1=-1\}, \\
& \{b 1=0, b 2=0, a 2=0, a 1=-1\}
\end{aligned}
$$

Using these results we can formulate the following

THEOREM 14. In the induced Toeplitz geometry all invariant (double) straight lines are exactly the following:

1., $x=z \quad y=z$

2., $\quad x=-z \quad y=-z$

3., $x=-z \quad y=0$

\section{Some Conclusions and Remarks}

Toeplitz has considered his matrices for the first time in 1901. Now they are ubiquitous and one of most well investigated classes of matrices. In the paper [8] is given big information for this. They can be applied in different fields of mathematics [7]. But nobody, as I know, has put the question when some subsets of these matrices are groups. So the notion Toeplitz Geometry comes for the first time in my papers. I want to express my thanks for the reviewer, who has make a useful recommendations

\section{REFERENCES}

[1] Stanilov G. and Sl. Slavova: Parallel between Circulate and Hankel Geometry, Proceedings of the International Conference, Shumen University, 2009, pp. 16-22.

[2] G. Stanilov: Even dimensional Circulate geometry, Results in Mathematics, Vol. 59, Nos.:3/4,2011,pp. $319-326$ 
[3] G. Stanilov: Toeplitz Geometry over vector Space induced from his 5-order Symmetric Matrices, Journal of Geometry, Vol.105, No.1m pp 201-202

[4] G. Stanilov: Geometry over 3-order symmetric Toeplitz matrices. Journal of Mathematics and System Science (accepted for publication).

[5] Hsuang-Chu Li: On Calculating the Determinants of Toeplitz Matrices, Journal of Applied Mathematics\&Bioinformatics,vol.1, 2011,55-64.

[6] Ke Ye, Lek-Heng Lim: Every Matrix is a Product of Toeplitz Matrices, arXiv.org>mat.>arxiv:1307.5132.2-13,[math.AG]. 